\title{
REMOVABLE EDGES ON A HAMILTON CYCLE OR OUTSIDE A CYCLE IN A 4-CONNECTED GRAPH ${ }^{1}$
}

\author{
Jichang $\mathrm{Wu}^{a}$, Hajo Broersma ${ }^{b}$, YaPing $\mathrm{MaO}^{c}$ \\ AND \\ QIN MA ${ }^{d, 2}$ \\ ${ }^{a}$ School of Mathematics \\ Shandong University, Jinan \\ Shandong 250100, China \\ ${ }^{b}$ Faculty of EEMCS, University of Twente \\ P.O. Box 217, 7500 AE Enschede, The Netherlands \\ ${ }^{c}$ Department of Mathematics \\ Qinghai Normal University \\ Xining, Qinghai 810008, China \\ ${ }^{d}$ Department of Biomedical Informatics \\ College of Medicine, Ohio State University \\ Columbus, OH, 43210, USA \\ e-mail: jichangwu@126.com \\ h.j.broersma@utwente.nl \\ maoyaping@ymail.com \\ qin.ma@osumc.edu
}

\begin{abstract}
Let $G$ be a 4-connected graph. We call an edge $e$ of $G$ removable if the following sequence of operations results in a 4-connected graph: delete $e$ from $G$; if there are vertices with degree 3 in $G-e$, then for each (of the at most two) such vertex $x$, delete $x$ from $G-e$ and turn the three neighbors of $x$ into a clique by adding any missing edges (avoiding multiple edges). In this paper, we continue the study on the distribution of removable edges in a 4-connected graph $G$, in particular outside a cycle of $G$ or in a spanning tree or on a Hamilton cycle of $G$. We give examples to show that our results are in some sense best possible.
\end{abstract}

Keywords: 4-connected graph, removable edge, fragment, atom.

2010 Mathematics Subject Classification: 05C40, 05C38, 05 C75.

\footnotetext{
${ }^{1}$ Research supported by China Scholarship Council (No. 201606225054).

${ }^{2}$ Corresponding author.
} 


\section{INTRODUCTION}

\section{REFERENCES}

[1] K. Ando, Y. Egawa, K. Kawarabayashi and M. Kriesell, On the number of 4contractible edges in 4-connected graphs, J. Combin. Theory Ser. B 99 (2009) 97-109. doi:10.1016/j.jctb.2008.04.003

[2] J.A. Bondy and U.S.R. Murty, Graph Theory (Springer Graduate Texts in Mathematics, 2008).

[3] D.A. Holton, B. Jackson, A. Saito and N.C. Wormald, Removable edges in 3-connected graphs, J. Graph Theory 14 (1990) 465-473.

doi:10.1002/jgt.3190140410

[4] P.J. Slater, A classification of 4-connected graphs, J. Combin. Theory Ser. B 17 (1974) 281-298. doi:10.1016/0095-8956(74)90034-3

[5] J.C. Wu, H.J. Broersma and H. Kang, Removable edges and chords of longest cycles in 3-connected graphs, Graphs Combin. 30 (2014) 743-753. doi:10.1007/s00373-013-1296-x

[6] J.C. Wu, X.L. Li and L.S. Wang, Removable edges in a cycle of a 4-connected graph, Discrete Math. 287 (2004) 103-111.

doi:10.1016/j.disc.2004.05.015

[7] J.C. Wu, X.L. Li and J.J. Su, The number of removable edges in a 4-connected graph, J. Combin. Theory Ser. B 92 (2004) 13-40.

doi:10.1016/j.jctb.2004.02.003

[8] J.H. Yin, Removable edges in 4-connected graphs and the structures of 4-connected graphs J. Systems Sci. Math. Sci. 19 (1999) 434-438.

Received 11 July 2018

Revised 23 December 2018

Accepted 9 February 2019 